Article

\title{
Preparation and Characterization of Chemically-Modified Biomaterials and Their Application as Adsorbents of Penicillin G
}

\author{
Jesie Silva ${ }^{1}$, Lizebel Morante ${ }^{1}$, Tesfamichael Demeke ${ }^{2}$, Jacqueline Baah-Twum ${ }^{2}$ \\ and Abel E. Navarro $2, *$ (D) \\ 1 Chemistry Section, Department of Exact Sciences, School of Sciences and Philosophy, \\ Cayetano Heredia University, Lima L31, Peru; jesie.silva@upch.pe (J.S.); lizebel.morante.a@upch.pe (L.M.) \\ 2 Science Department, Borough of Manhattan Community College, City University of New York, NY 10007, \\ USA; tesfademeke@hotmail.com (T.D.); baahjacqueline94@gmail.com (J.B.-T.) \\ * Correspondence: anavarro@bmcc.cuny.edu; Tel.: +1-212-220-8000
}

Received: 22 May 2018; Accepted: 30 August 2018; Published: 5 September 2018

\begin{abstract}
The prevalence of antibiotics in water creates microbial resistance and has a negative impact on the ecosystem. Biomaterials such as spent tea leaves are rich in functional groups and are suitable for chemical modification for diverse applications. This research proposes the use of spent tea leaves of chamomile (CM), green tea (GT), and peppermint (PM) as structural scaffolds for the incorporation of carboxyl, sulfonyl, and thiol groups to improve the adsorption of Penicillin $G$ $(\mathrm{Pe})$. Adsorbents characterization reported a higher number of acidic functional groups, mainly in thiolated products. Scanning electron microscopy (SEM) analysis showed changes on the surfaces of the adsorbents due to reaction conditions, with a stronger effect on thiolated and sulfonated adsorbents. Elemental analysis by Energy dispersive X-ray spectrophotometry (EDS) corroborated the chemical modification by the presence of sulfur atoms and the increase in oxygen/carbon ratios. Batch experiments at different $\mathrm{pH}$ shows a strong $\mathrm{pH}$-dependence with a high adsorption at $\mathrm{pH} 8$ for all the adsorbents. The adsorption follows the trend CMs > GTs $>$ PMs. Thiolation and sulfonation reported higher adsorptions, which is most likely due to the sulfur bridge formation, reaching adsorption percentages of $25 \%$. These results create a new mindset in the use of spent tea leaves and their chemical modifications for the bioremediation of antibiotics.
\end{abstract}

Keywords: adsorption; thiolation; carboxylation; sulfonation; spent tea leaves; Penicillin G (Pe)

\section{Introduction}

It is widely known that more than $70 \%$ of the world's surface is covered by the hydrosphere, in which life and Earth's morphologies depend on. Moreover, water is involved in one of the most vital chemical reactions, photosynthesis, which is the basis of life on our planet [1]. It has been estimated that the water volume in the world is distributed as $97.3 \%$ seawater, found in seas and oceans, and $2.7 \%$ as freshwater, which can also be divided into $2.04 \%$ as ice caps and glaciers and the remaining $0.7 \%$ is represented by surface and underground water. In conclusion, only $0.7 \%$ of the total water in the world can be directly utilized for human consumption [2,3].

This small percentage of drinkable water is not only used for human consumption, but also for industrial processes and for the rest of the biota. Unfortunately, since the Industrial Revolution, certain substances have been discharged into surface water, making it unsuitable for consumption. These substances mainly include heavy metals, dyes and organic compounds that derive from crude oil [4]. Therefore, constant efforts have been developed to eliminate and/or recover these substances from water. These techniques include electrodeposition, reverse osmosis, 
photodegradation, microprecipitation, the use of activated sludge, adsorption, ultracentrifugation, ozonolysis, and biodegradation [4,5]. Among these techniques, adsorption has reported the most positive results, due to its low-cost, easy applicability, the recovery of the adsorbates, and the recycling of the adsorbents [5]. Even though activated carbon is known to be the most powerful adsorbent for the decontamination of air and water [4-6], it still shows drawbacks, including its activation and thermal treatment that involves the release of carbon dioxide into the atmosphere. This $\mathrm{CO}_{2}$ has an effect on the greenhouse effect in the long-term range [6]. Recent efforts have been invested in the use of naturally-occurring materials, such as marine algae, products from seashells, and lignocellulosic materials in their raw state as potential adsorbents of water pollutants [7-10]. For example, hydrogel beads of chitosan and alginate have been used in the removal of caffeine [11], even in the presence of salts and other interfering substances. Moreover, 2-chlorophenol was adsorbed from aqueous solutions by raw spent tea leaves, reaching an adsorption percentage of $80 \%$ [12]. Further, recently, raw and conditioned spent tea leaves were utilized for the desalination of water with positive results [13]. These prior researches indicated that the biopolymers that are present in these materials are rich in organic functional groups such as hydroxyl, carbonyl, carboxyl, amine, ester, amide, and others. Apparently, heavy metal ions and polar pollutants have a preference towards carboxyl and highly polar groups on the adsorbent. Therefore, our new direction points towards the chemical modification of raw spent tea leaves as a platform for the incorporation of carboxyl, thiol and sulfonyl groups to enhance the adsorptive properties of these biomaterials [11-13].

On the other hand, the growth of the economy and migration of population to the great metropolis has created new habits on the personal care in our society, which are generating new emerging contaminants. These include pharmaceutical and personal care products. These species did not pose any toxicity, but with their constant use, their concentration levels are reaching values that cause negative impacts on human health and the ecosystem. To humans, the presence of antibiotics in water increases the probability of antibiotic resistance, decreasing the potency of our current drugs to fight those resistant microbes. To the ecosystem, these antibiotics have a negative impact on the availability of innocuous aquatic microbes that are essential to the trophic chain of those ecosystems. Due to the recent identification of these emerging contaminants, there is no legal regulation that is able to determine the maximum allowable concentrations in the environment [14,15]. The most representative pharmaceutical products that have been detected in water, and that are pharmacologically active, include: Anti-inflammatory and analgesics; anti-depressors; anti-epileptic; hypolipidaemic drugs; $\beta$-inhibitors; antihistaminic; and antibiotics [16].

Therefore, this research aims to develop new chemically-modified adsorbents from spent tea leaves to enhance their adsorptive properties towards the antibiotic Penicillin $\mathrm{G}(\mathrm{Pe})$. This antibiotic was chosen due to its widespread use in human health to treat gonorrhea, salmonella, diphtheria, E. coli infections, among others, and its commercial availability; even without medical prescription in some developing countries. A practical chemical modification methodology of spent tea leaves is described, as well as the characterization of the modified adsorbents and the preliminary adsorption tests of Pe.

\section{Experiments}

\subsection{Conditioning of the Adsorbents}

Chamomile (CM), green tea (GT), and peppermint (PM) teabags were obtained from a local market. Other teabags come as a mixture of spices and herbs, which is not ideal for an adsorbent or chemical modification [12]. Tea samples were vigorously boiled in tap water to eliminate impurities that attribute color, smell and taste to the tea leaves. Final rinses were done with boiling distilled and deionized water to eliminate any chemical residues such as salts and other water-soluble substances. Then, the water-boiled tea samples were oven-dried at no more than $50{ }^{\circ} \mathrm{C}$, to prevent heat decomposition, for at least one day. Finally, tea samples were cut open, leaves were crushed, sieved to a particle 
diameter between $100 \mu \mathrm{m}$ and $200 \mu \mathrm{m}$, and stored in plastic containers at room temperature. Since the final goal was to chemically-modify the adsorbents, it was mandatory to eliminate any substance from the cellulosic structure to assure that the chemical modification occurs on the cellulose and not on these poorly-soluble species (i.e., chlorophyll, pigments and/or substances that are not highly soluble in water). To accomplish this, the samples were passed through a continuous extraction with the Soxhlet technique. Tea samples underwent solvent extraction with a 1:1 mixture of ethanol/acetone during $24 \mathrm{~h}$. Adsorbents looked less colored (light brown for GT and PM, and pale yellow for CM), confirming the elimination of these undesired species. Upon continuous solvent extraction, the samples were again oven-dried at a temperature no higher than $50{ }^{\circ} \mathrm{C}$ overnight, and stored in plastic containers at room temperature until their use for the chemical modification, or for the control adsorption tests. It is important to highlight that these conditioned adsorbents did not undergo any spoiling or biological degradation for the entire duration of the study (more than 4 months), as demonstrated by electron microscopy and infrared techniques (data not shown). Stability tests were run as checkpoints to demonstrate there is no change of adsorption capacities over time.

\subsection{Chemical Modification of the Adsorbents}

Carboxyl, thiol and sulfonyl groups were incorporated to the cellulosic structures of CM, GT and PM by adapting the procedures from previously reported studies [17-19]. These chemical modifications were adjusted to our tea leaf samples, since it is expected that a more complex mixture of biopolymers, and not pure cellulose or fibers as indicated in these researches.

The carboxylation was adapted from Wang et al. [17], who used carboxylated bamboo fibers for the adsorption of lead (II) ions. In brief, our methodology was as follows: $10 \mathrm{~g}$ of conditioned biomass was mixed with $40 \mathrm{~g}$ of citric acid (Reagent grade, Fisher Scientific, Atlanta, GA, USA), sodium hypophosphite (Reagent grade, Fisher Scientific) and enough distilled water to make a suspension. The mixture was sonicated at room temperature during $20 \mathrm{~min}$. Then, the suspension was stirred during $24 \mathrm{~h}$ at $55^{\circ} \mathrm{C}$. After, the temperature was increased to $125^{\circ} \mathrm{C}$ during $90 \mathrm{~min}$, also under stirring. Lastly, the suspension was washed with distilled water until the rinses were neutral. The carboxylated adsorbents were oven dried, crushed, sieved $(100 \mu \mathrm{m}>\mathrm{d}>200 \mu \mathrm{m})$ and stored in plastic container until used. The carboxylated samples were labeled as CCM, CGT and CPM for chamomile, GT and peppermint, respectively.

The methodology of sulfonation by Liimatainen et al. [18] was utilized and adapted to our adsorbents. $10 \mathrm{~g}$ of the conditioned adsorbent was soaked in $330 \mathrm{~mL}$ of distilled water in an Erlenmeyer flask. Then, $2.7 \mathrm{~g}$ of sodium periodate (Reagent grade, Fisher Scientific) and sodium bisulfite were mixed and stirred at a temperature of $55^{\circ} \mathrm{C}$ for $3 \mathrm{~h}$. Upon this initial stirring, the suspension was left under stirring during 3 days at room temperature. Then, the samples were washed with distilled water until the rinses were neutral. The sulfonated adsorbents were oven dried, crushed, sieved $(100 \mu \mathrm{m}>d>200 \mu \mathrm{m})$ and stored in plastic containers until used. The sulfonated samples were labeled as SCM, SGT and SPM for chamomile, GT and peppermint, respectively.

$\mathrm{Wu}$ et al. [19] thiolated cellulosic biomass for the removal of lead (II) ions. This technique was modified for our tea leaf samples as follows: $20 \mathrm{~g}$ of tea leaves were suspended in $690 \mathrm{~mL}$ of distilled water and mixed with $10 \mathrm{~mL}$ of mercaptoacetic acid (Reagent grade, Fisher Scientific) under a well-ventilated hood. The mixture was placed under stirring at room temperature during $24 \mathrm{~h}$. Then, the thiolated samples were oven dried, crushed, sieved $(100 \mu \mathrm{m}>d>200 \mu \mathrm{m})$ and stored in plastic containers until used. The thiolated samples were labeled as TCM, TGT and TPM for chamomile, green tea and peppermint, respectively.

\subsection{Preparation and Quantification of Penicillin G Solutions}

Stock solutions of 1000 ppm (mg/L) were daily prepared from Pe Potassium salt (Analytical grade, Fisher Scientific) and used without further purification. Stock solutions were made by dissolving the antibiotic with deionized water by reverse osmosis, with a conductivity of $18 \mathrm{~m} \Omega$. 
Then, the stock solution was kept in glass containers and stored under refrigeration within the $24 \mathrm{~h}$. Adsorption experiments were conducted by dilution of the stock solution until reaching the desired concentration. Initial, and at equilibrium concentration, of Pe in the solution was quantified by ultraviolet spectrophotometry with a microplate reader (Synergy 4, Biotek, Winooski, VT, USA) at a wavelength of $220 \mathrm{~nm}$ [20]. Glassware was carefully washed and rinsed with deionized water to prevent any biological contamination by external sources.

\subsection{Adsorption Experiments}

Discontinuous assays were carried out at room temperature in an orbital shaker during $12 \mathrm{~h}$ (according to preliminary runs). Experiments were conducted in triplicates and the reported results were obtained by averaging all the values. Batch experiments were always performed with a blank sample (without adsorbent) to compare the concentrations of Pe, before and after exposure to the adsorbents. Polyethylene plastic tubes were used for the discontinuous tests and were sealed to avoid any leakage. Plastic tubes did not display any interaction with $\mathrm{Pe}$, as demonstrated by preliminary experiments. The water that was used for the dilution of the stock solution as well as the adsorbents was autoclaved before each experiment to prevent any bacterial growth that could lead to a decrease in the Pe concentration. Initial solution $\mathrm{pH}$ was studied and compared the efficiency of the native and chemically-modified adsorbents. The initial solution $\mathrm{pH}$ ranged from two to eight and was adjusted to the required value by adding aliquots of diluted $\mathrm{HCl}$ and $\mathrm{NaOH}$ to the solutions prior to contact with the adsorbents. $50 \mathrm{mg}$ of the adsorbents were suspended in $50 \mathrm{~mL}$ of Pe solutions with a concentration of $50 \mathrm{ppm}$. The $\mathrm{pH}$ effect has been considered as the most important experimental parameter on the adsorption of pollutants [7-12] and was chosen in this study to compare the adsorptive properties of the 12 adsorbents.

\subsection{Characterization of the Adsorbents}

The surface properties as well as texture and morphology of the native and chemically-modified adsorbents were observed by Scanning Electron Microscopy (SEM), using a Table Top Microscopy, TM3000 (Hitachi, Tarrytown, NY, USA). No gold-coating was utilized to observe the samples. Elemental analysis of the adsorbents was simultaneously studied by Energy Dispersive X-Ray Spectrophotometry (EDS) with a Bruker spectrometer (Quantax 70, Tarrytown, NY, USA). This technique allows us to determine the percent abundance of key elements that are associated with the chemical modification, including an increase of oxygen (for carboxylation) or the presence of sulfur (for sulfonation and thiolation). Lastly, the chemical modifications increase the number of acidic functional groups (thiol, carboxyl and sulfonyl). These groups can be quantified by volumetric titration of the adsorbents and expressed as concentration of carboxyl groups as described elsewhere [21]. For this test, $5 \mathrm{~g}$ of the native and chemically-modified adsorbents were suspended in a $0.1 \mathrm{M}$ solution of sodium nitrate (to keep a constant ionic strength) and titrated against a standardized sodium hydroxide solution to determine the concentration of acidic groups on the adsorbents.

\subsection{Data Analysis of the Adsorption Experiments}

Quantification of Pe was expressed as adsorption percentage (\%ADS), as described by Equation (1):

$$
\% \text { ADS }=\frac{\left(C_{i}-C_{e q}\right)}{C_{i}} \times 100
$$

where $C_{i}$ and $C_{e q}$ are the initial and at equilibrium concentrations of Pe in the solution. Mathematical and statistical treatment was performed using Excel (Microsoft Office) and Origin v5.0 (OriginLab). Errors were calculated and ranged between $3.5-4.0 \%$ in all the experiments. Error bars are not shown in the plots in order to avoid crowding of curves. 


\section{Results and Discussion}

\subsection{Chemical Modification of the Adsorbents}

Antibiotics are complex organic compounds with a diverse range of functional groups. Pe, shown in Figure 1, includes polar functional groups like carboxyl, amide, and other more hydrophobic, such as aromatic ring and thioether. Based on prior work, dyes, phenols and other organic pollutants tend to adsorb onto biomasses through ionic exchange or hydrogen bonding [7-12], indicating that the uptake of pollutants could be enhanced by the addition of more or new polar functional groups to the surface of the biomass. Likewise, $\mathrm{pH}$ plays an important role on adsorption, due to the chemical speciation and ionization of adsorbate and adsorbent in aqueous solution [5,6]. These scenarios prompted us to incorporate polar and ionizable functional groups to our previously used adsorbents to enhance the adsorption of pollutants of emerging concern, such as Pe. Carboxyl and sulfonyl were chosen due to their high polarity and $\mathrm{pH}$-dependence, whereas thiol groups were selected based on their polarity and because thiols have reported high affinity towards other types of pollutants such as heavy metals $[5,6,19]$.

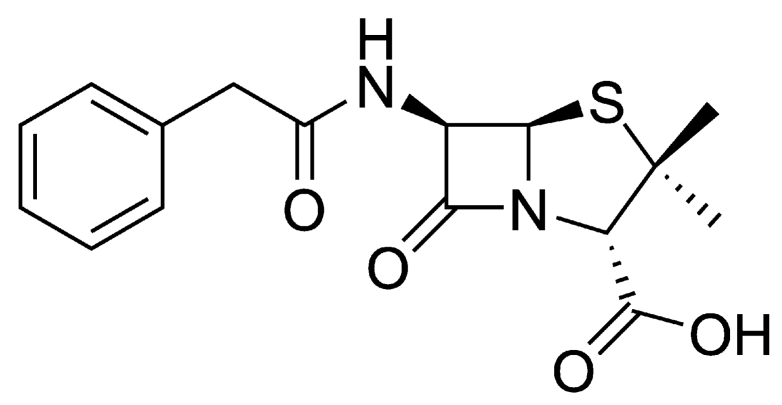

Figure 1. Chemical structure of Penicillin G (Pe).

Our approach involves the use of spent tea leaves, which are disposed by tea-based companies such as Nestea, Snapple and others, who need to take care of these solid residues upon the brewing process. GT, PM and CM were used as model lignocellulosic materials because they are composed of one type of tea, therefore the adsorption properties will be only be attributed to that type of tea leaf. Other commercially available teas come as a blend with cinnamon, cloves, and adsorption might display deviations or abnormalities. These spent tea leaves were used as scaffolds for the addition of carboxyl, sulfonyl and thiol groups. Our chemical modification was totally carried out in an aqueous systems, in the absence of organic solvents, following a Green Chemistry mindset. No heavy metals or highly toxic organic compounds are used during the syntheses. While these new adsorbents report a higher adsorption affinity towards Pe than the native adsorbents, they involve an extra expense associated with the conditioning and the chemical modification. At this moment, our research is focusing on the optimization of the chemical reaction with the reduction of chemicals, by mechanical steps, such as sonication, stirring, temperature gradients, and other types of ligno-cellulosic materials. For this research, CM, GT and PM were chemically modified according to the reaction schemes displayed in Figure 2. As observed in the Figure, the chemical modifications involved practical reactions, including Fisher esterification (carboxylation and thiolation) and diol cleavage followed by nucleophilic attack (sulfonation). 

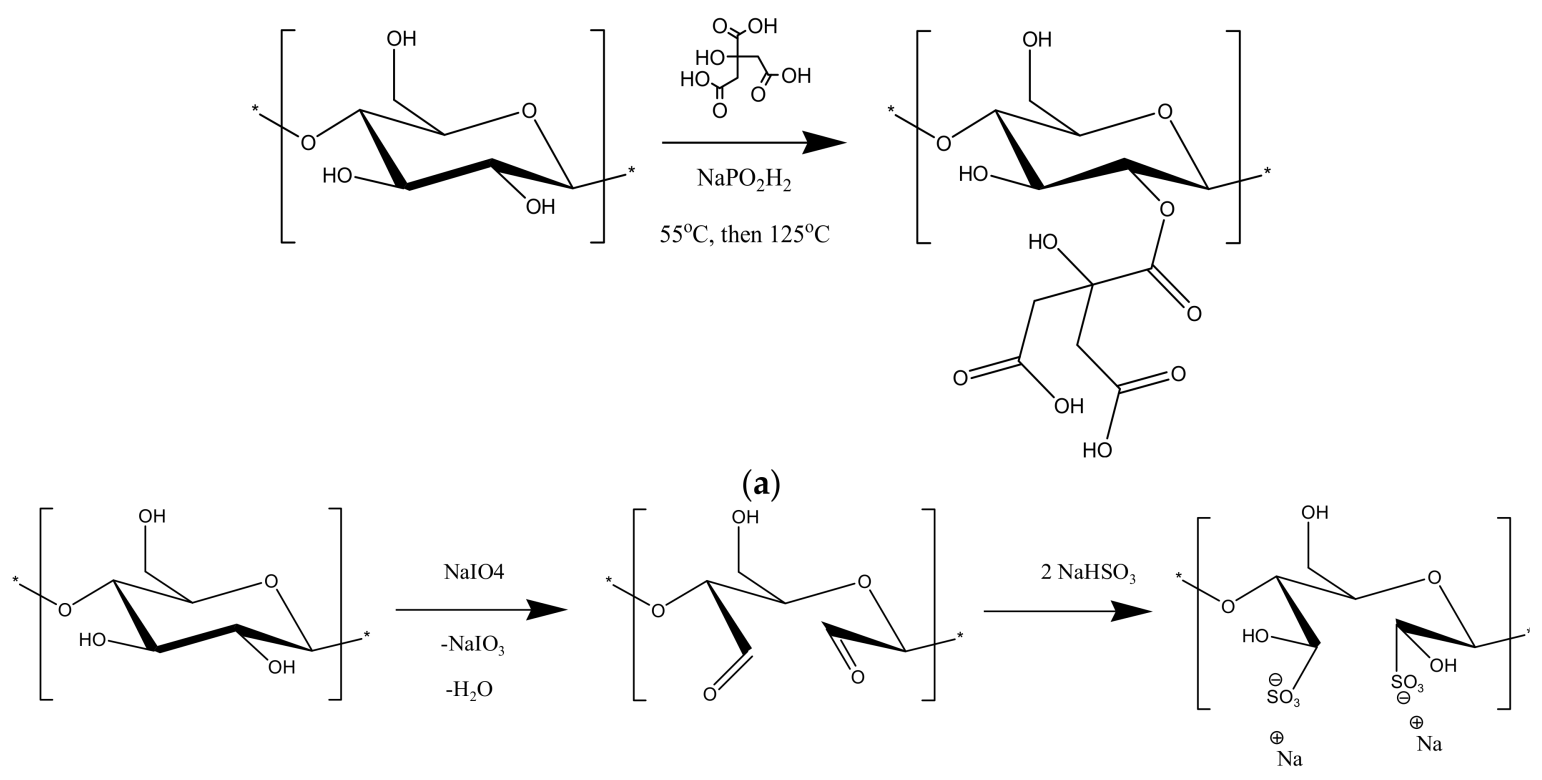

(b)
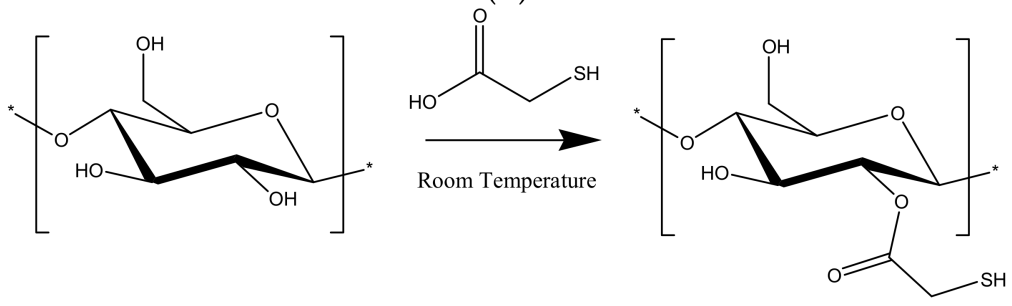

(c)

Figure 2. Scheme with the chemical modifications: (a) carboxylation; (b) sulfonation; and (c) thiolation.

\subsection{Characterization of the Adsorbents}

The chemically-modified adsorbents were characterized by classical and instrumental techniques to determine their chemical, morphological and textural properties. All the adsorbents were volumetrically titrated against $\mathrm{NaOH}$ to determine the concentration of acidic groups (as carboxyl groups) and the data analysis followed the procedure as reported elsewhere [21]. The results are shown in Table 1, with the amount of $-\mathrm{COOH}$ groups present in each adsorbent. According to the results, all the chemical modification resulted in an increase in the amount of acidic groups, indicating the incorporation of new groups in their structures. Since all the chemical modification sacrifice hydroxyl groups from the glucose units, the increase in the number of acidic groups could be attributed to the incorporation of the new functional groups.

Table 1. Quantification of ionizable acidic groups of native and chemically-modified adsorbents.

\begin{tabular}{cccccc}
\hline Adsorbent & $\mathrm{C}_{\mathrm{COOH}}(\mathbf{m m o l} / \mathbf{g})$ & Adsorbent & $\mathrm{C}_{\mathrm{COOH}}(\mathbf{m m o l} / \mathbf{g})$ & Adsorbent & $\mathbf{C}_{\mathrm{COOH}}(\mathbf{m m o l} / \mathbf{g})$ \\
\hline $\mathrm{CM}$ & 1.36 & $\mathrm{GT}$ & 1.72 & $\mathrm{PM}$ & 1.4 \\
$\mathrm{CCM}$ & 1.48 & $\mathrm{CGT}$ & 1.88 & $\mathrm{CPM}$ & 2 \\
$\mathrm{SCM}$ & 1.76 & $\mathrm{SGT}$ & 2.02 & $\mathrm{SPM}$ & 1.58 \\
$\mathrm{TCM}$ & 1.98 & $\mathrm{TGT}$ & 2.08 & $\mathrm{TPM}$ & 1.66 \\
\hline
\end{tabular}

The data also indicates that CM and GT offer structures that are friendlier towards thiolation, as noted by the highest change in acidic groups. Conversely, PM displays a higher number of acidic groups for carboxylation. Overall, all the native adsorbents increased the number of acidic groups. 
As mentioned before, it is expected to increase the magnitude of the incorporation of the chemical modifications with the changes that are being currently studied.

The textural and morphological changes of all nine adsorbents were also explored by SEM. The three types of tea underwent similar changes with the chemical modifications, but the most noticeable results were observed in chamomile. The micrographs are displayed in Figure 3 for $\mathrm{CM}$ and their chemical modifications. As shown by the images, there is a substantial change on the surface of the adsorbent before and after the chemical reactions. However, they all show the same pattern, characterized by a heterogeneous surface, with the presence of vessels, pockets and protrusions that are ideal in adsorbents. These caves and pockets serve as traps for the antibiotic by the exposure of more functional groups that are able to interact with the antibiotic and prevent their return into the bulk solution. It is important to highlight that even though all the adsorbents show structural damages, the thiolation seems to have a more intense impact on the surface, followed by the sulfonation and carboxylation. This could be explained by the chemical reaction that occurs for each modification. Thiolation consists on the exposure of the tea leaves in mercaptoacetic acid. It is expected to observe certain degradation of the cellulose under acidic conditions. Apparently, some cellulose strands are hydrolyzed in the presence of this organic acid. On the other hand, sulfonation consists on the mild oxidation of diols with sodium periodate. This oxidation could potentially lead to deterioration of the cellulose structure, as shown in the micrographs. However, this effect is not as intense as in thiolation. Finally, carboxylation displays the mildest effect on the surface of $\mathrm{CM}$, indicating that citric acid is not as aggressive as mercaptoacetic acid or sodium periodate. A plausible explanation for this observation is the presence of sodium hypophosphite, which acts as an esterification catalyst and might reduce the acidity of the reaction mixture, preventing the hydrolysis of the cellulose chains.

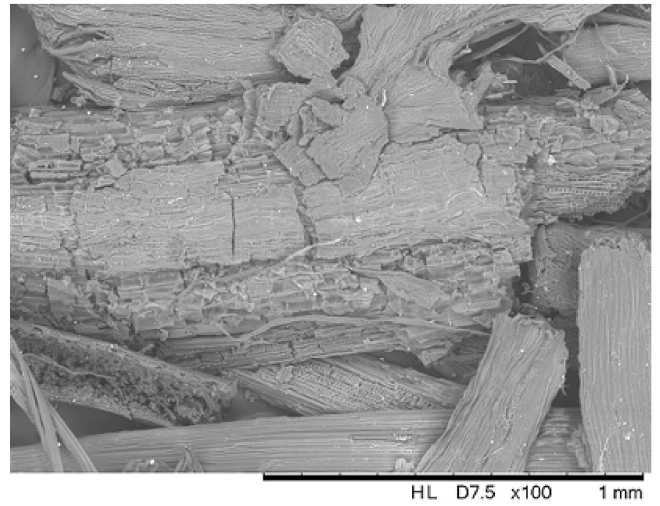

(a)

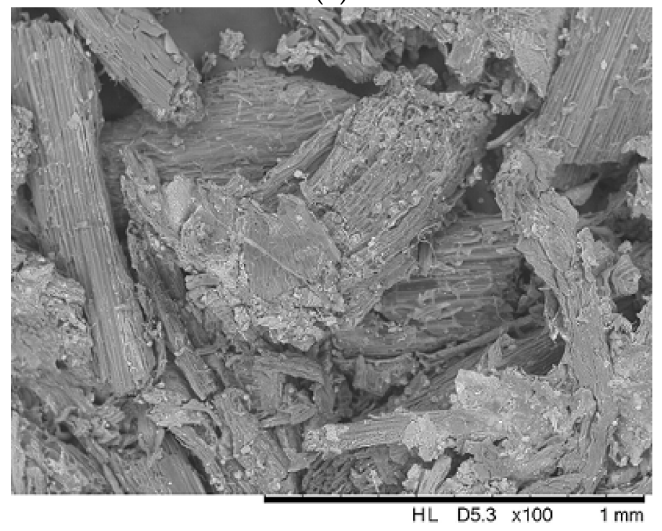

(c)

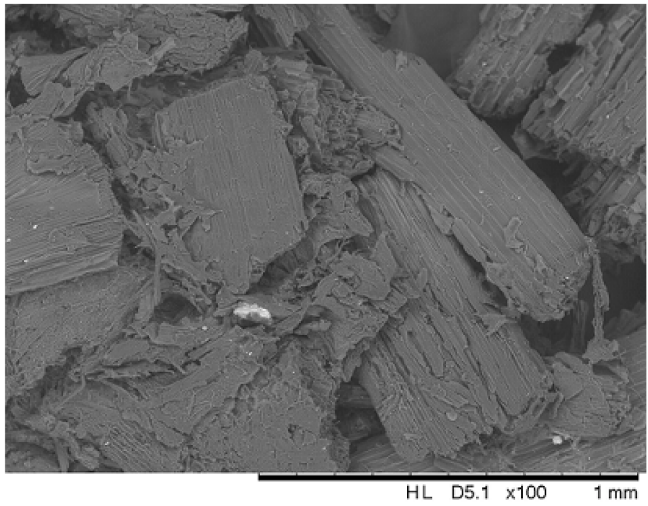

(b)

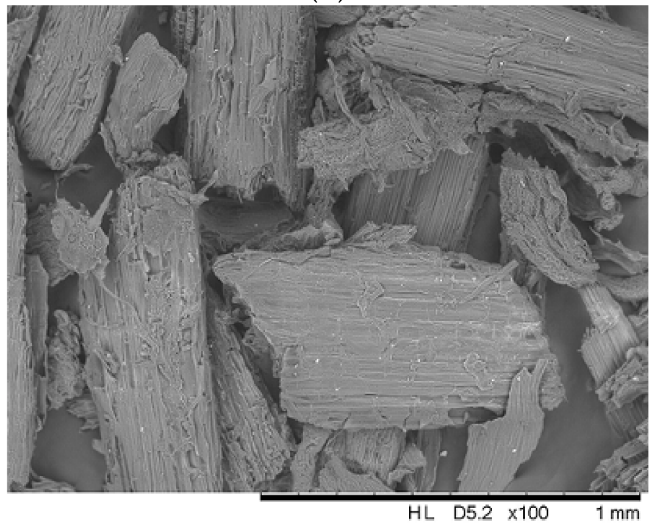

(d)

Figure 3. Scanning electron micrographs of CM adsorbent series: (a) Native CM; (b) CCM; (c) SCM; and (d) TCM at $100 \times$ magnification. 
Finally, the elemental composition of the adsorbents were investigated by EDS and the results are displayed in Figure 4 and Table 2. According to the results, there is no substantial change in the elemental composition of the adsorbents, with carbon and oxygen as the most predominant species. This is expected since the chemical modifications were carried out on cellulose as a scaffold and therefore the most abundant structure in the material. However, Table 2 shows the relative element abundances as quantified by X-rays. As observed in the data, sulfur was successfully added to SCM and TCM, with the respective decrease in carbon and oxygen percentages. However, as discussed before, a damage on the cellulose structure is confirmed by the decrease on carbon content for all the adsorbent, and with lower intensity in the carboxylated adsorbent. Moreover, carboxylation shows a higher oxygen/carbon content ratio in respect to the native adsorbent, indicating the higher oxygen content due to the addition of carboxyl groups. Sulfonation also shows a higher oxygen/carbon ratio because sulfonyl groups also contain oxygen atoms. Lastly, the results also show a higher sulfur content in TCM than in SCM, this could be associated with the efficiency of the thiolation reaction compared to the sulfonation. This data corroborates the number of acidic groups of SCM and TCM (1.76 and $1.98 \mathrm{mmol} / \mathrm{g}$, respectively). Lastly, fluorine is observed in some of the EDS spectra in agreement with recent findings that confirm the presence of fluoride ions in tea leaves [22]. Apparently, tea plants have a high affinity towards fluoride and readily absorb it from the soil.

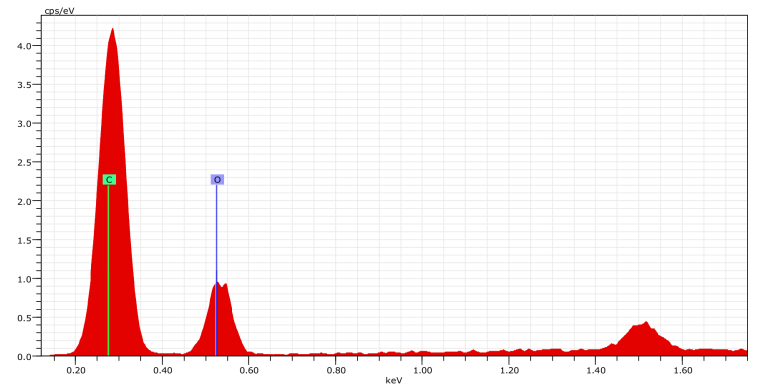

(a)

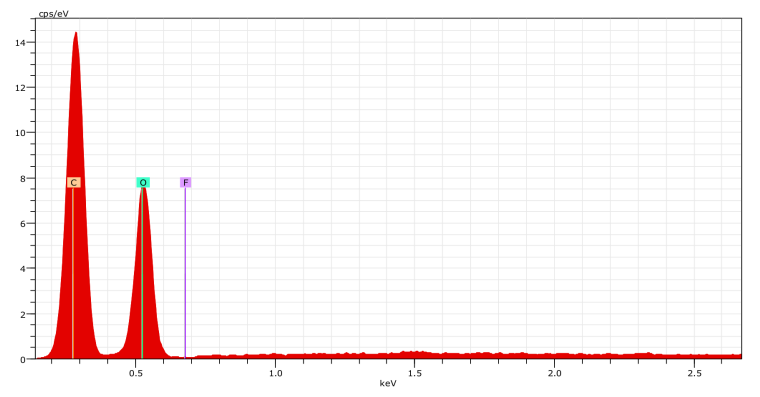

(c)

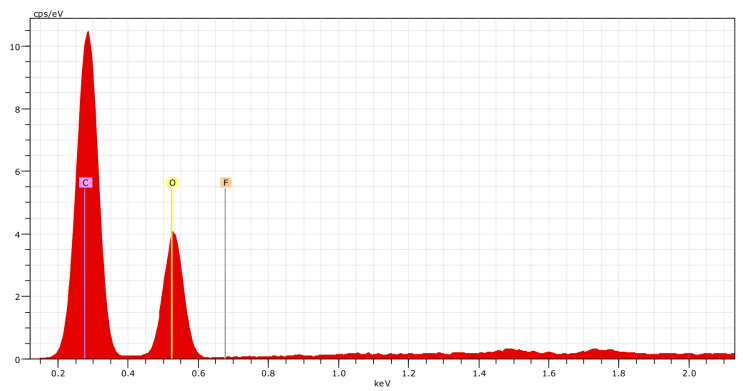

(b)

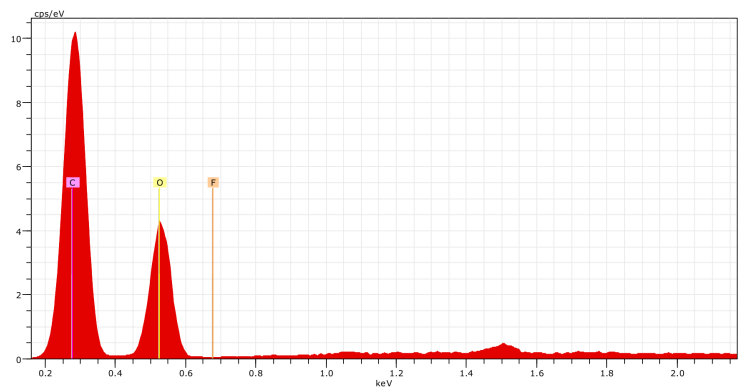

(d)

Figure 4. Energy dispersive X-ray spectrophotometry (EDS) analysis of the CM adsorbent series: (a) Native CM; (b) CCM; (c) SCM; and (d) TCM. Sulfur was detected but not visible at the graph scale.

Table 2. Element percentages of key elements of the CM adsorbent series by EDS analysis.

\begin{tabular}{cccc}
\hline Adsorbent & \% Carbon & \% Oxygen & \% Sulfur \\
\hline CM & 72.07 & 27.93 & 0 \\
CCM & 65.12 & 34.89 & 0 \\
SCM & 58.92 & 39 & 2.08 \\
TCM & 62.69 & 35.11 & 2.20 \\
\hline
\end{tabular}




\subsection{Effect of the Initial Solution $p H$}

Batch adsorption assays were conducted with the native and chemically-modified adsorbents to evaluate their adsorption efficiency towards $\mathrm{Pe}$ at changing $\mathrm{pH}$ values and explores the potential application of these materials as adsorbents of antibiotics. The $\mathrm{pH}$ ranged from two to eight to resemble surface water and industrial wastewaters that are commonly in the acidic range. The results were divided by the adsorbent series and are shown in Figure 5. From the graphs, it could be concluded that $\mathrm{CM}$ series are the best adsorbents before and after chemical modifications (\%ADS range between 20-25\%). These results do not match the previously reported data [7-10], where PM had the highest affinity towards heavy metals. This could be explained by the nature of antibiotics, as complex organic molecules, whose interaction no longer depends on a single atom (or metal ion), but to the force average of the polar and hydrophobic interactions that are present in Pe. PM series are next with $\%$ ADS around $16-18 \%$ and then GT adsorbents with 15-16\%. It is important to highlight that even though these \% ADS values do not resemble a complete removal of Pe from solutions, but it is more than evident in the effect of the chemical modification on the uptake of Pe. Currently, our group is conducting adsorption optimization tests to increase the adsorption percentage of these biomaterials (unpublished work).

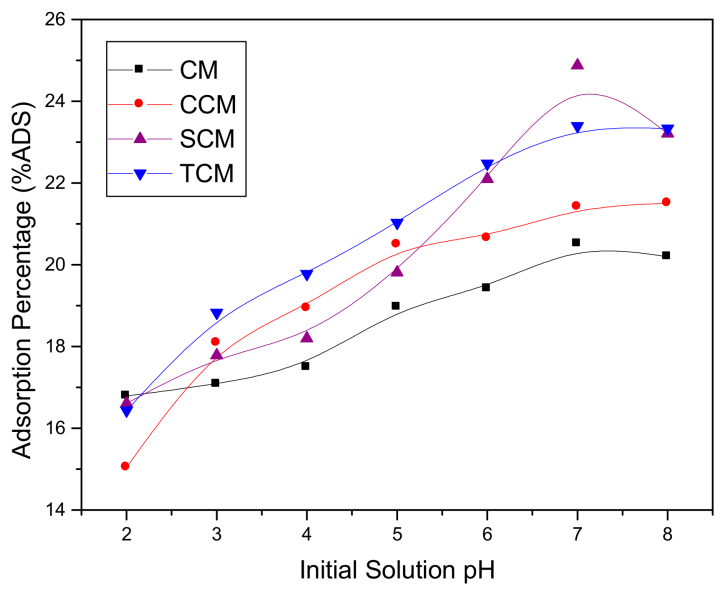

(a)

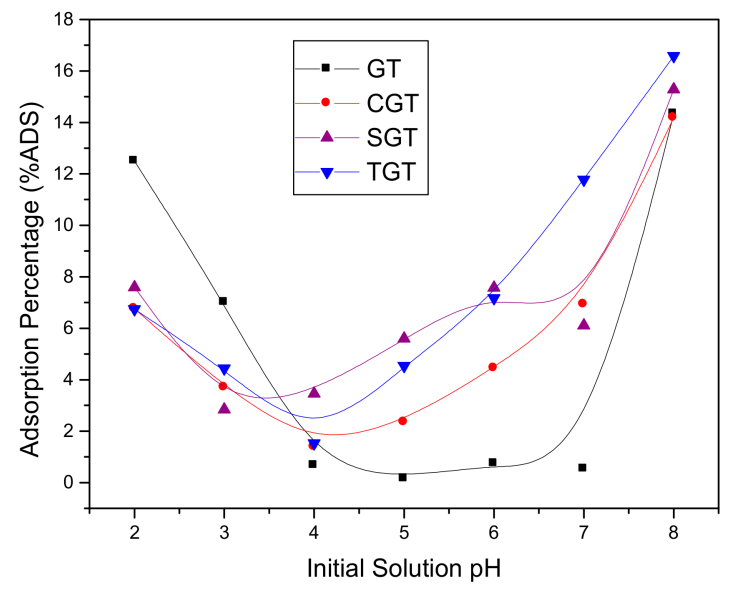

(b)

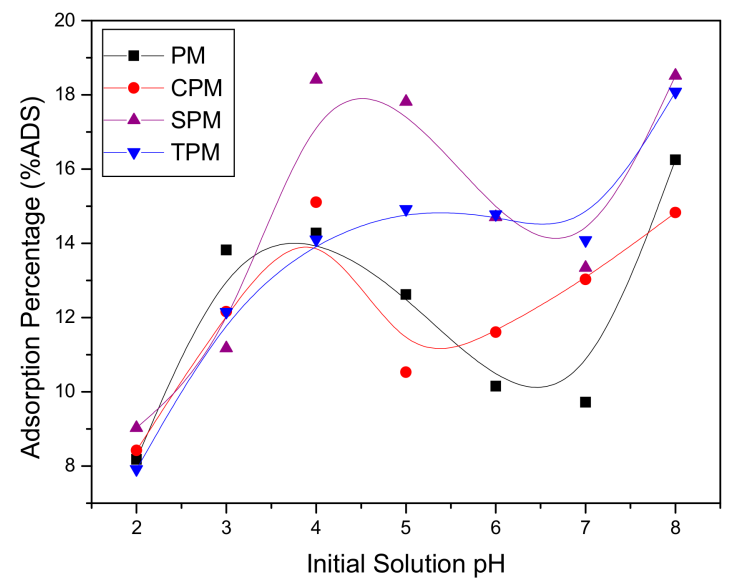

(c)

Figure 5. Effect of the $\mathrm{pH}$ on the adsorption of Pe onto native and chemically-modified tea leaves:

(a) CM adsorbent series; (b) GT adsorbent series; and (c) PM adsorbent series.

The importance of this preliminary test resides on the affinity enhancement of the adsorbents towards Pe. The adsorption trends were as follows: SCM > TCM > CCM > CM, for the GT series it was 
TGT $>$ SGT $>$ CGT $\geq$ GT and for the PM adsorbents it was SPM $>$ TPM $>$ PM $>$ CPM. In conclusion, the presence of sulfur functional groups increase the uptake of Pe. Conversely, carboxylation does not seem to have an effect, in fact, for PM, the carboxylation reported an adsorption that is even lower than the native adsorbent. It could be hypothesized that since Pe also has a carboxyl group (Figure 1), the carboxylated adsorbents repels Pe due to same-charge incompatibility. This same charge is produced due to the high solution $\mathrm{pH}$ at which the adsorption occurs. On the other hand, thiolated adsorbents could be interacting with Pe at the sulfur atoms level by the formation of sulfide bridge or stronger dipoles forces due to the presence of sulfur. A similar effect could be proposed for sulfonation, whose adsorption percentage is slightly lower than in the thiolated version, apparently due to the repulsion of the carboxyl Pe with the negatively charged sulfonates.

It is also important to notice that even though the SEM studies indicate a damage on the surfaces of the materials, their adsorption percentage still remains above the native adsorbents. This demonstrates that the chemical modifications have a positive effect on these biomaterials, which are even able to balance and surpass the deterioration of the morphological and textural properties of the adsorbents. Our current work includes the optimization of the chemical modification techniques to maximize the incorporation of functional groups with the minimum damage of the structures of the adsorbents.

\section{Conclusions}

The development of the pharmaceutical and personal care products has increased with the world population. The presence of these species jeopardizes human health and the ecosystem. Alternative and naturally-occurring adsorbents from spent leaves were used as structural scaffolds for the incorporation of carboxyl, sulfonyl and thiol functional groups, which have proven a higher affinity towards pollutants from solutions. This study reports the chemical modification and material characterization of the resulting adsorbents from chamomile, GT and peppermint spent tea leaves and their preliminary application for the removal of Pe from solutions. The number of acidic functional groups were determined, indicating the addition of these groups to the tea samples. Data indicates that thiolation occurs at a higher rate than the other chemical modifications. Morphological studies by SEM showed a deterioration on the surface of the adsorbent, mainly due to the experimental conditions that were used during the reactions, including oxidants heat and acid exposure. EDS, corroborated the presence of the functional groups by the presence of sulfur atoms and higher oxygen/carbon ratios, when compared to the native adsorbents. Finally, the antibiotic adsorption was tested at different $\mathrm{pH}$ values to evaluate their application on the removal of Pe. Results indicate that sulfur plays a major role on the uptake of Pe, due to a possible formation of sulfide bridge. Carboxylation displayed the lowest effect, most likely due to same-charge repulsion at high solution $\mathrm{pH}$. This research will definitely open new avenues on the use of biological wastes such as spent tea leaves as structural scaffolds for chemical modification and their use in environmental remediation.

Author Contributions: J.S. and L.M. prepared the adsorbents and optimized the synthetic methodology and conducted preliminary adsorption tests. T.D. and J.B.-T. conducted the material characterization and carried out the $\mathrm{pH}$ effect on the adsorption. A.E.N. supervised the work and structure the research.

Funding: This research was funded by INNOVATE-Peru grant number 169-PNCIP-ECIP-2015 to AEN and the 2017 BMCC Faculty Development Grant to AEN.

Acknowledgments: Bertha Llanos (R.I.P.) is greatly acknowledged for her support and for her guidance on this research project and personally as a chemist.

Conflicts of Interest: The authors declare no conflict of interest.

\section{References}

1. Cunningham, V.; Buzby, M.; Hutchinson, T.; Mastrocco, F.; Parke, N.; Roden, N. Effects of Human Pharmaceuticals on Aquatic Life: Next Steps. Environ. Sci. Technol. 2006, 40, 3456-3462. [CrossRef] [PubMed] 
2. Jjemba, P. Excretion and Ecotoxicity of Pharmaceutical and Personal Care Products in the Environment. Ecotoxicol. Environ. Saf. 2006, 63, 113-130. [CrossRef] [PubMed]

3. Ternes, T.; Joss, A. Human Pharmaceuticals, Hormones and Fragrances: The Micropollutant Challenge for Urban Water Management; IWA Publishing: London, UK, 2006; Volume 21, p. 53.

4. Liu, Y.; Wang, J. Fundamentals and Applications of Biosorption Isotherms, Kinetics and Thermodynamics; Nova Science Publishers: New York, NY, USA, 2009.

5. Kotrba, P.; Machova, M.; Macek, T. Microbial Biosorption of Metals; Springer Science: New York, NY, USA, 2011.

6. Volesky, B. Sorption and Biosorption; BV SORBEX Inc.: Quebec, QC, Canada, 2004.

7. Choi, Y.; Isaac, P.; Irkakhujaev, S.; Masud, M.E.; Navarro, A.E. Use of spent tea wastes-chitosan capsules for the removal of divalent copper ions. J. Environ. Sci. 2015, 1, 003.

8. Park, R.; Kim, G.; Shen, L.; Hong, M.; Navarro, A.E. Batch adsorption of heavy metals onto chai tea residues for the bioremediation of contaminated solutions. Curr. Top. Biotechnol. 2014, 8, 51-62.

9. Navarro, A.E.; Musaev, H.; Serrano, K.; Masud, M.E. Adsorption kinetics of cobalt (II) ions onto alginate beads from aqueous solutions. J. Earth Sci. Clim. Chang. 2014, 5, 223. [CrossRef]

10. Zahir, H.; Naidoo, M.; Kostadinova, R.M.; Ortiz, K.A.; Sun-Kou, R.; Navarro, A.E. Decolorization of hair dye by lignocellulosic waste materials from contaminated waters. Front. Environ. Toxicol. 2014, 2, 28. [CrossRef]

11. Zarzar, A.; Hong, M.; Llanos, B.; Navarro, A.E. Insights into the eco-friendly adsorption of caffeine from contaminated solutions by using hydrogel beads. J. Environ. Anal. Chem. 2015, 2, 4.

12. Japhe, T.; Zhdanova, K.; Rodenburg, L.; Roberson, L.; Navarro, A.E. Factors affecting the Biosorption of 2-Chlorophenol using spent tea leaf wastes as adsorbents. J. Environ. Sci. 2015, 1, 010.

13. Kim, G.; Garcia, H.; Japhe, T.; Llanos, B.; Navarro, A.E. On the desalination of saline waters via batch adsorption with spent tea leaves. J. Pet. Environ. Biotechnol. 2017, 8, 3.

14. Richardson, M.; Bowron, J. The Fate of Pharmaceutical Chemicals in the Aquatic Environment. J. Pharmacy Pharmacol. 1985, 37, 1-12. [CrossRef]

15. Fent, K.; Weston, A.; Caminada, D. Ecotoxicology of Human Pharmaceuticals. Aquat. Toxicol. 2006, 76, 122. [CrossRef] [PubMed]

16. Bush, K. Antimicrobial Agents. Curr. Opin. Chem. Biol. 1997, 1, 169-175. [CrossRef]

17. Wang, S.; Wang, L.; Kong, W.; Ren, J.; Liu, C.; Wang, K.; Sun, R.; She, D. Preparation, characterization of carboxylated bamboo fibers and their adsorption for lead(II) ions in aqueous solution. Cellulose 2013, 20, 2091-2100. [CrossRef]

18. Liimatainen, H.; Visanko, M.; Sirvio, J.; Hormi, O.; Niinimaki, J. Sulfonated cellulose nanofibrils obtained from wood pulp through regioselective oxidative bisulfite pre-treatment. Cellulose 2013, 20, 741-749. [CrossRef]

19. Wu, Z.; Cheng, Z.; Ma, W. Adsorption of $\mathrm{Pb}(\mathrm{II})$ from glucose solution on thiol-functionalized cellulosic biomass. Bioresour. Technol. 2012, 104, 807-809. [CrossRef] [PubMed]

20. Japhe, T.; Paulsingh, R.; Ko, K.; Hong, J.; Navarro, A.E. Bioremoval of antibiotics by using biodegradable hydrogel beads from aqueous solutions. J. Environ. Sci. 2015, 1, 002.

21. Navarro, A.E.; Portales, R.; Sun-Kou, R.; Llanos, B. Effect of pH on phenol biosorption by seaweeds. J. Hazard. Mater. 2008, 156, 405-411. [CrossRef] [PubMed]

22. Minton, B. Is Your Tea Loaded with Toxic Fluoride? Available online: https://naturalsociety.com/is-yourtea-loaded-with-toxic-fluoride/ (accessed on 10 May 2017).

(c) 2018 by the authors. Licensee MDPI, Basel, Switzerland. This article is an open access article distributed under the terms and conditions of the Creative Commons Attribution (CC BY) license (http://creativecommons.org/licenses/by/4.0/). 